\title{
A Flexible Approach Toward Tri-Substituted Piperidines and Indolizidines: Synthesis of 6-epi-Indolizidine 223A
}

\author{
Joel M. Harris and Albert Padwa* \\ Department of Chemistry, Emory University, Atlanta GA 30322 \\ chemap@emory.edu
}

Supporting Information Available: ${ }^{1} \mathrm{H}$ and ${ }^{13} \mathrm{C}-\mathrm{NMR}$ spectra for new compounds lacking elemental analyses together with an Ortep of compound 15. This material is available free of charge via the Internet at http://pubs.acs.org.

\section{General Experimental Section Paragraph}

Melting points are uncorrected. Mass spectra were determined at an ionizing voltage of $70 \mathrm{eV}$. Unless otherwise noted, all reactions were performed in flame dried glassware under an atmosphere of dry argon. Solutions were evaporated under reduced pressure with a rotary evaporator and the residue was chromatographed on a silica gel column using a 5\% ethyl acetate/hexane mixture as the eluent unless specified otherwise. All solids were recrystallized from $3 \%$ ethyl acetate/hexane for analytical data.
Table of Contents for Supporting Information
Page S2
${ }^{1} \mathrm{H}-\mathrm{NMR}$ spectrum of compound $\mathbf{1 7}$
Page S3
${ }^{1} \mathrm{H}-\mathrm{NMR}$ spectrum of compound $\mathbf{1 3}$
Page S4
${ }^{1} \mathrm{H}-\mathrm{NMR}$ spectrum of compound $\mathbf{1 8}$
Page S5
${ }^{1} \mathrm{H}-\mathrm{NMR}$ spectrum of compound $\mathbf{1 9}$
Page S6
${ }^{1} \mathrm{H}-\mathrm{NMR}$ spectrum of compound $\mathbf{2 0}$
Page S7
${ }^{1} \mathrm{H}-\mathrm{NMR}$ spectrum of compound $\mathbf{2 2}$
Page S8
${ }^{1} \mathrm{H}-\mathrm{NMR}$ spectrum of compound $\mathbf{2 3}$
Page S9
${ }^{13} \mathrm{C}-\mathrm{NMR}$ spectrum of compound $\mathbf{2 4}$
Page S10
${ }^{1} \mathrm{H}-\mathrm{NMR}$ spectrum of compound $\mathbf{2 5}$
Page S11
${ }^{1} \mathrm{H}-\mathrm{NMR}$ spectrum of compound $\mathbf{2 6}$
Page S12
${ }^{1} \mathrm{H}-\mathrm{NMR}$ spectrum of compound $\mathbf{2 7}$
Page S13
${ }^{1} \mathrm{H}-\mathrm{NMR}$ spectrum of compound 28
Page S14
${ }^{1} \mathrm{H}-\mathrm{NMR}$ spectrum of Benzhydrylidene-[4-(tert-butyldiphenyl- silanyloxy)-1-furan-2-yl-butyl] amine
Page S15 $\quad{ }^{1}$ H-NMR spectrum of 4-(tert-Butyl-diphenyl-silanyloxy)-1-furan -2-yl-butylamine.
Page S16 $\quad{ }^{1} \mathrm{H}-\mathrm{NMR}$ spectrum of compound $\mathbf{3 2}$
Page S17 $\quad{ }^{1} \mathrm{H}-\mathrm{NMR}$ spectrum of compound $\mathbf{3 3}$
Page S18 ${ }^{1} \mathrm{H}-\mathrm{NMR}$ spectrum of compound $\mathbf{3 4}$
Page S19 $\quad{ }^{1} \mathrm{H}-\mathrm{NMR}$ spectrum of compound $\mathbf{3 5}$
Page S20 $\quad{ }^{1} \mathrm{H}-\mathrm{NMR}$ spectrum of compound $\mathbf{3 6}$
Page S21 $\quad{ }^{1} \mathrm{H}$-NMR spectrum of compound $\mathbf{3 7}$
Page S22 $\quad{ }^{1}$ H-NMR spectrum of compound 39
Page S23 $\quad{ }^{1} \mathrm{H}-\mathrm{NMR}$ spectrum of compound $\mathbf{5 a}$
Page S24 $\quad{ }^{13}$ C-NMR spectrum of compound 5a
Page S25 Ortep Drawing of compound 15 


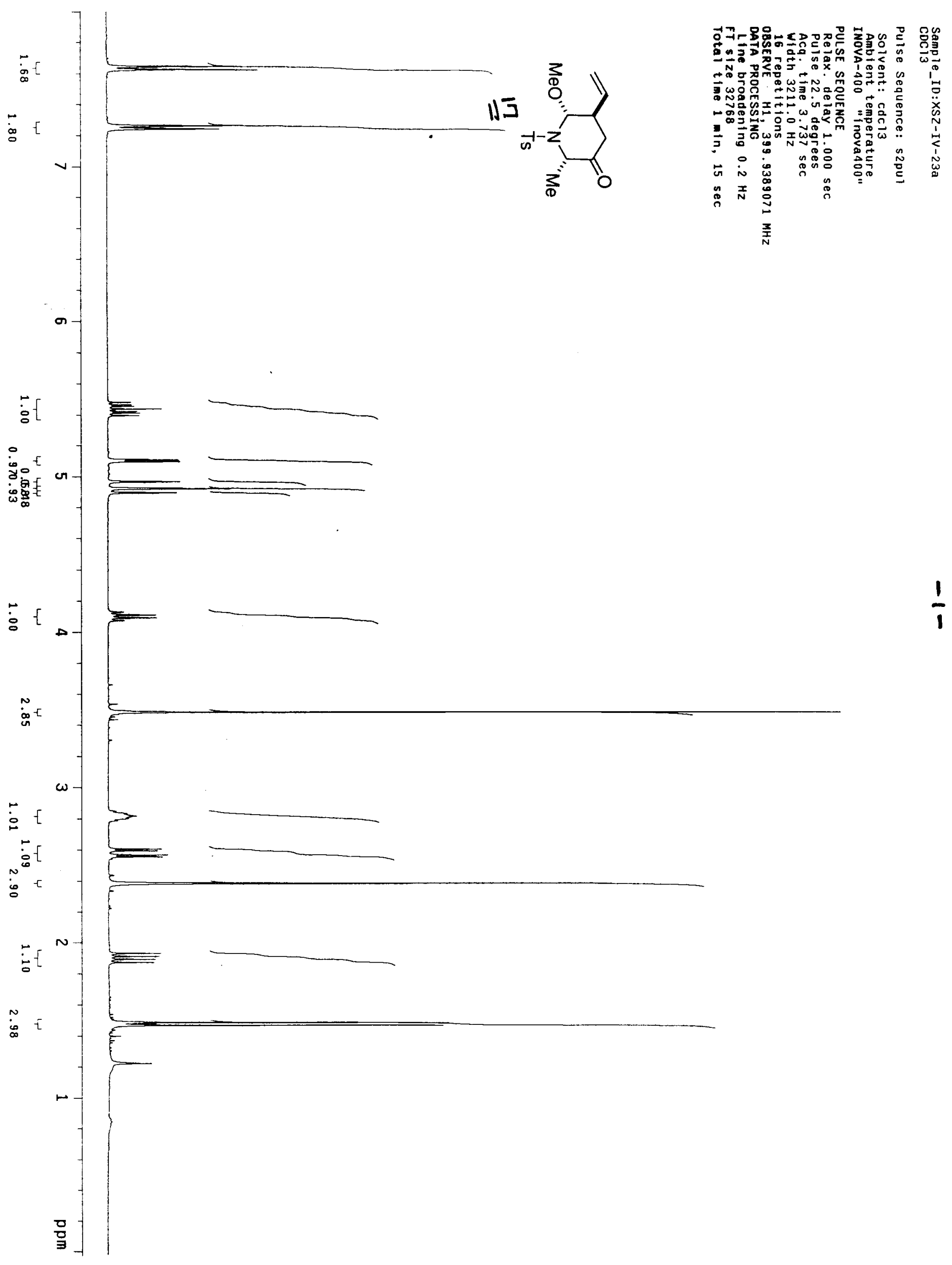




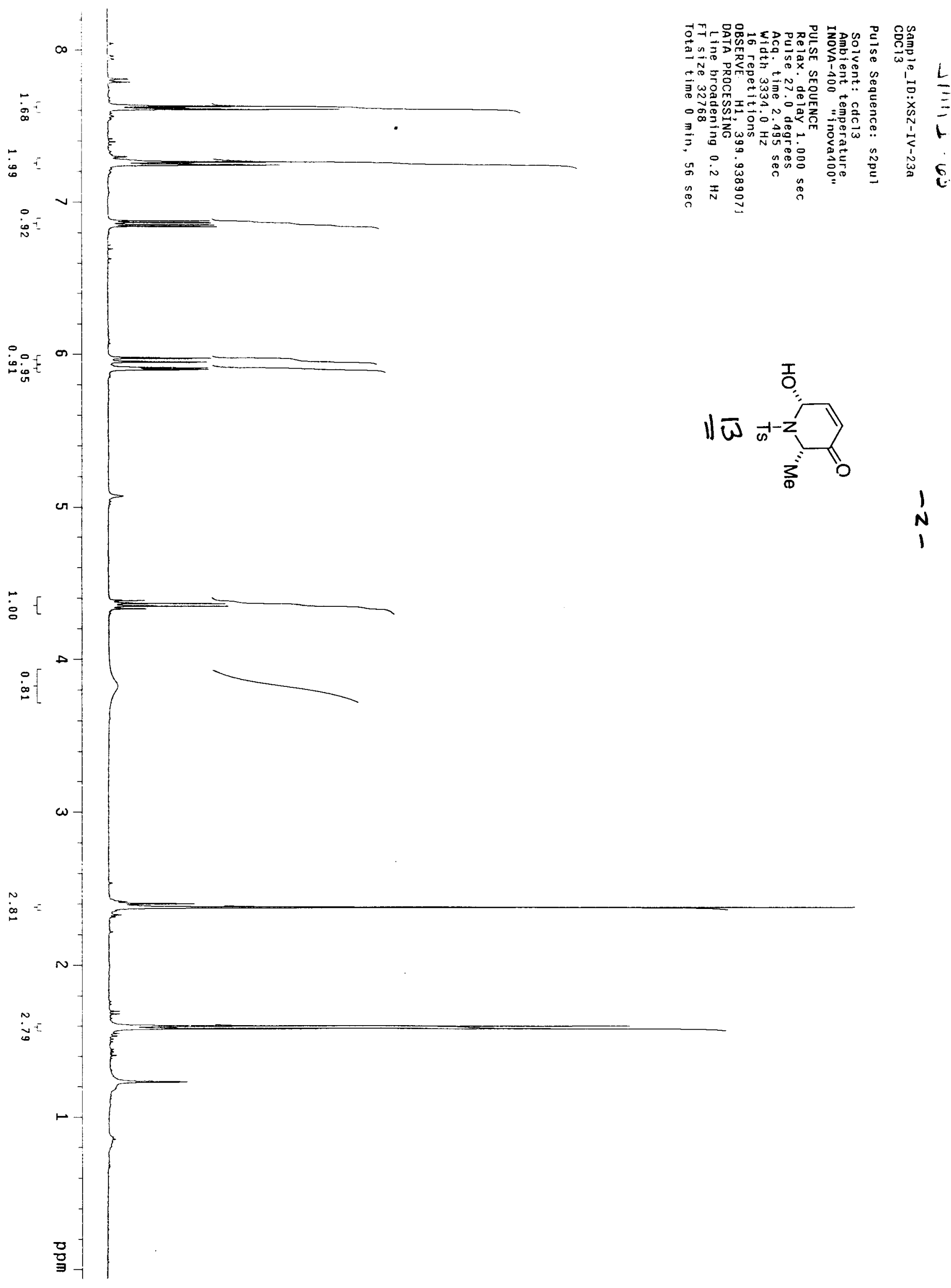




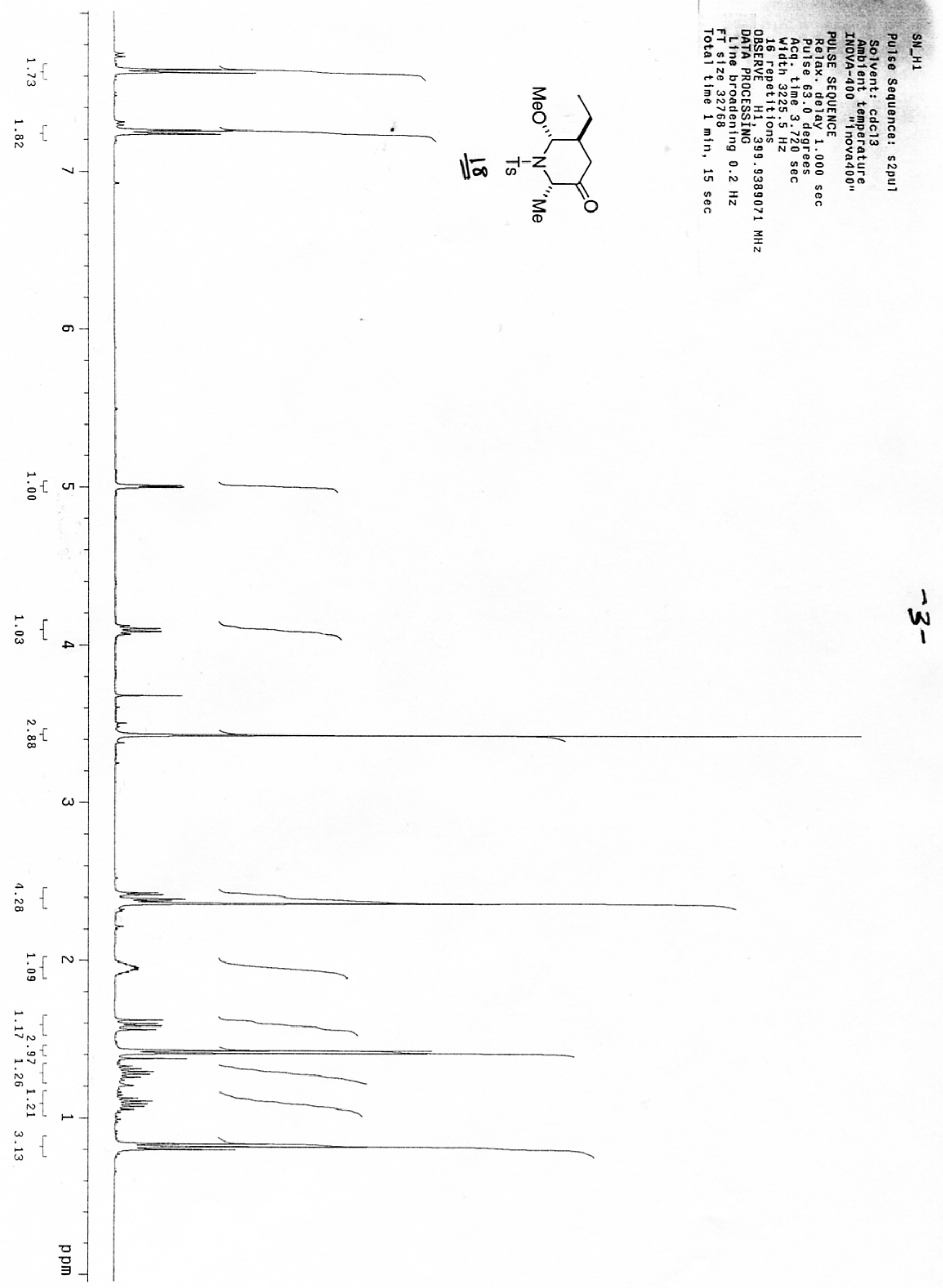




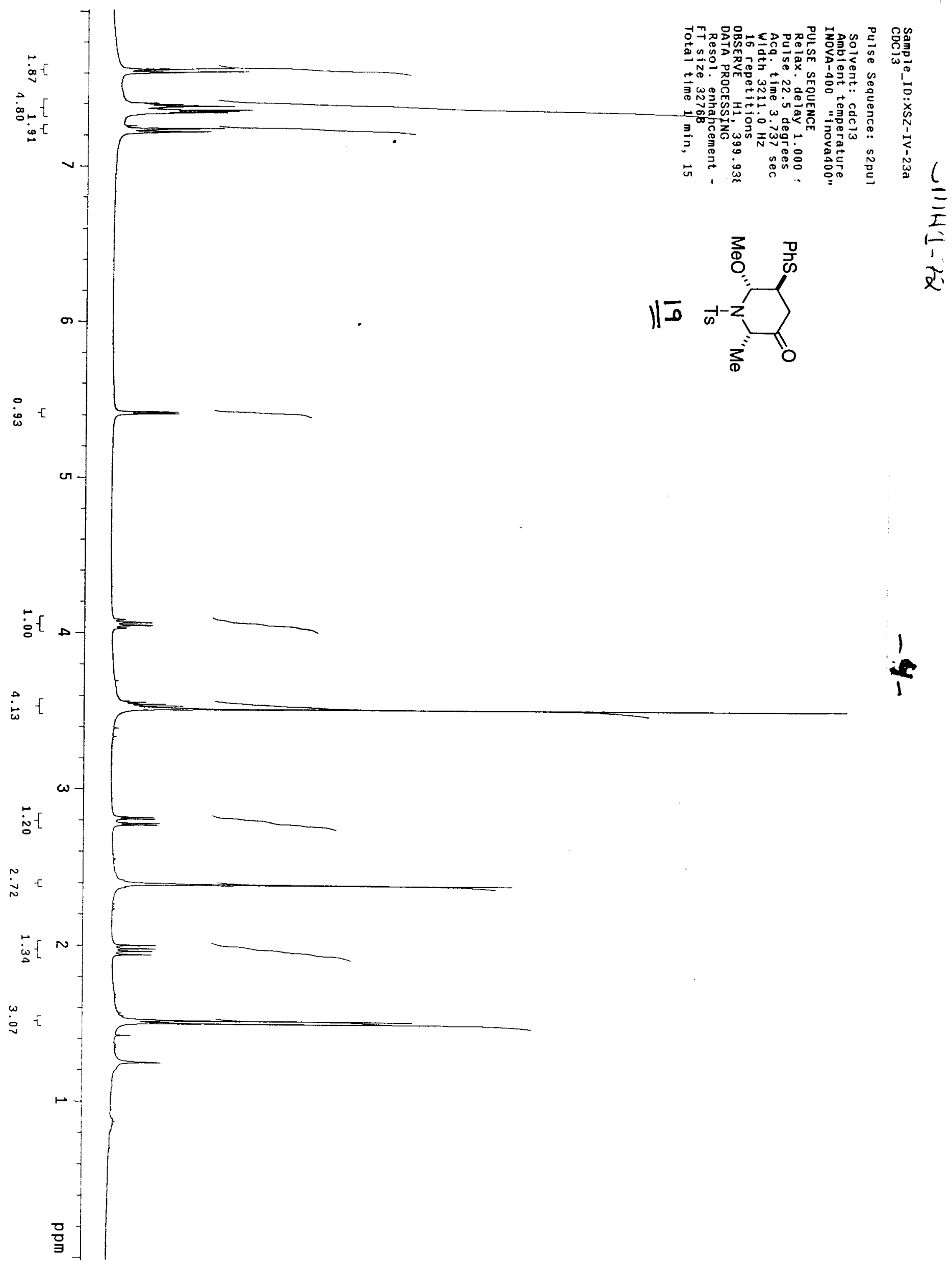




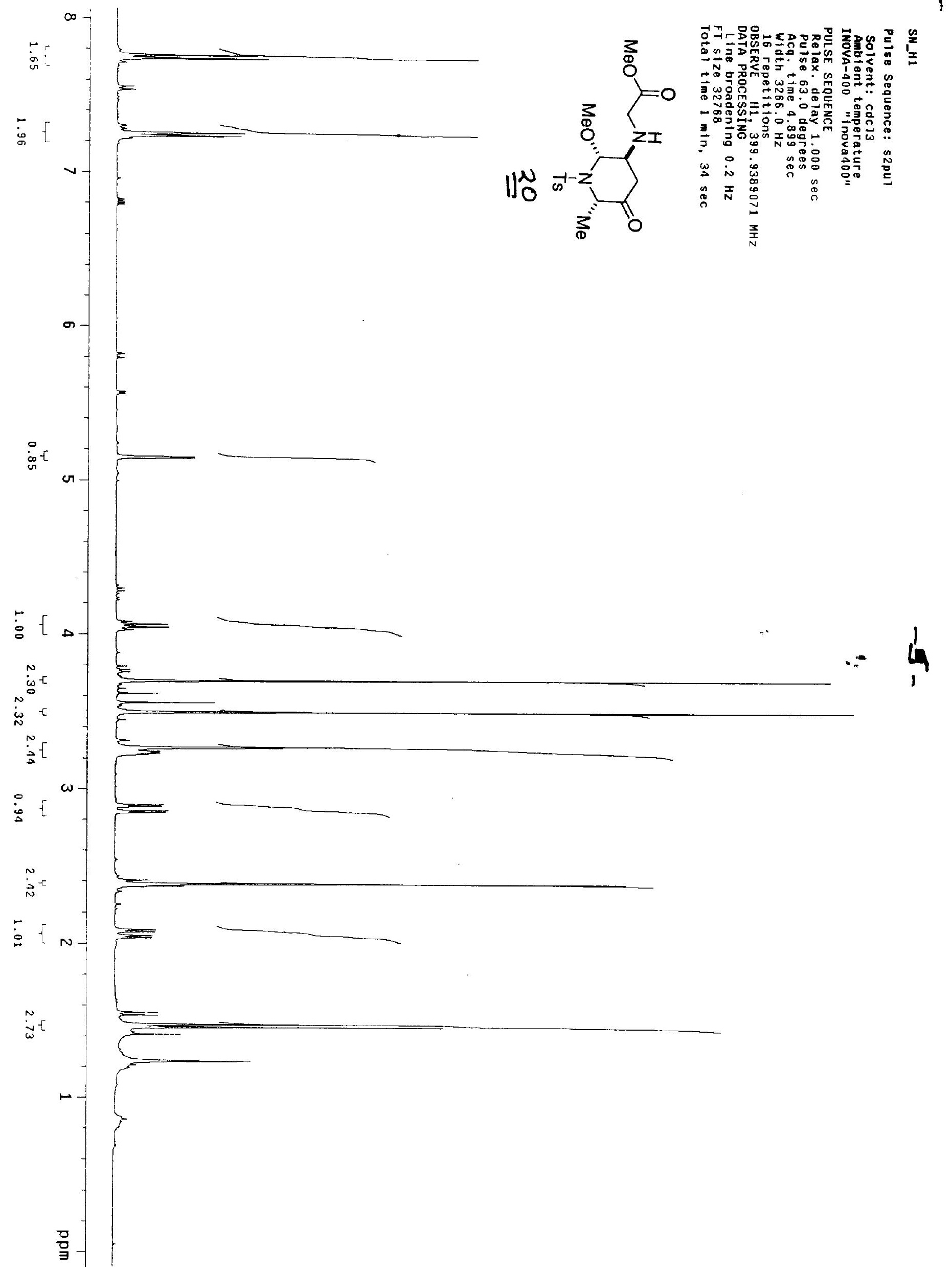




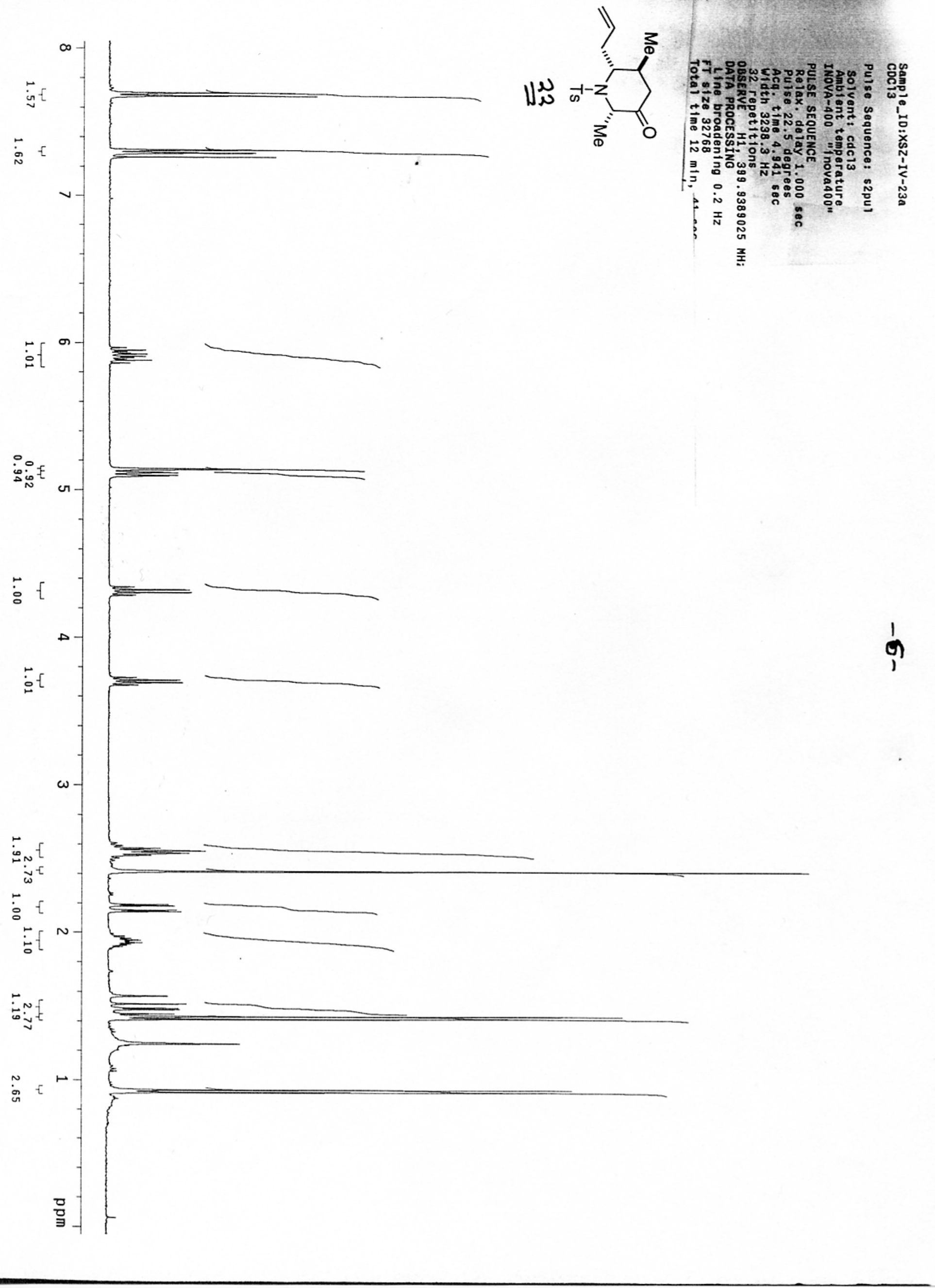


ร
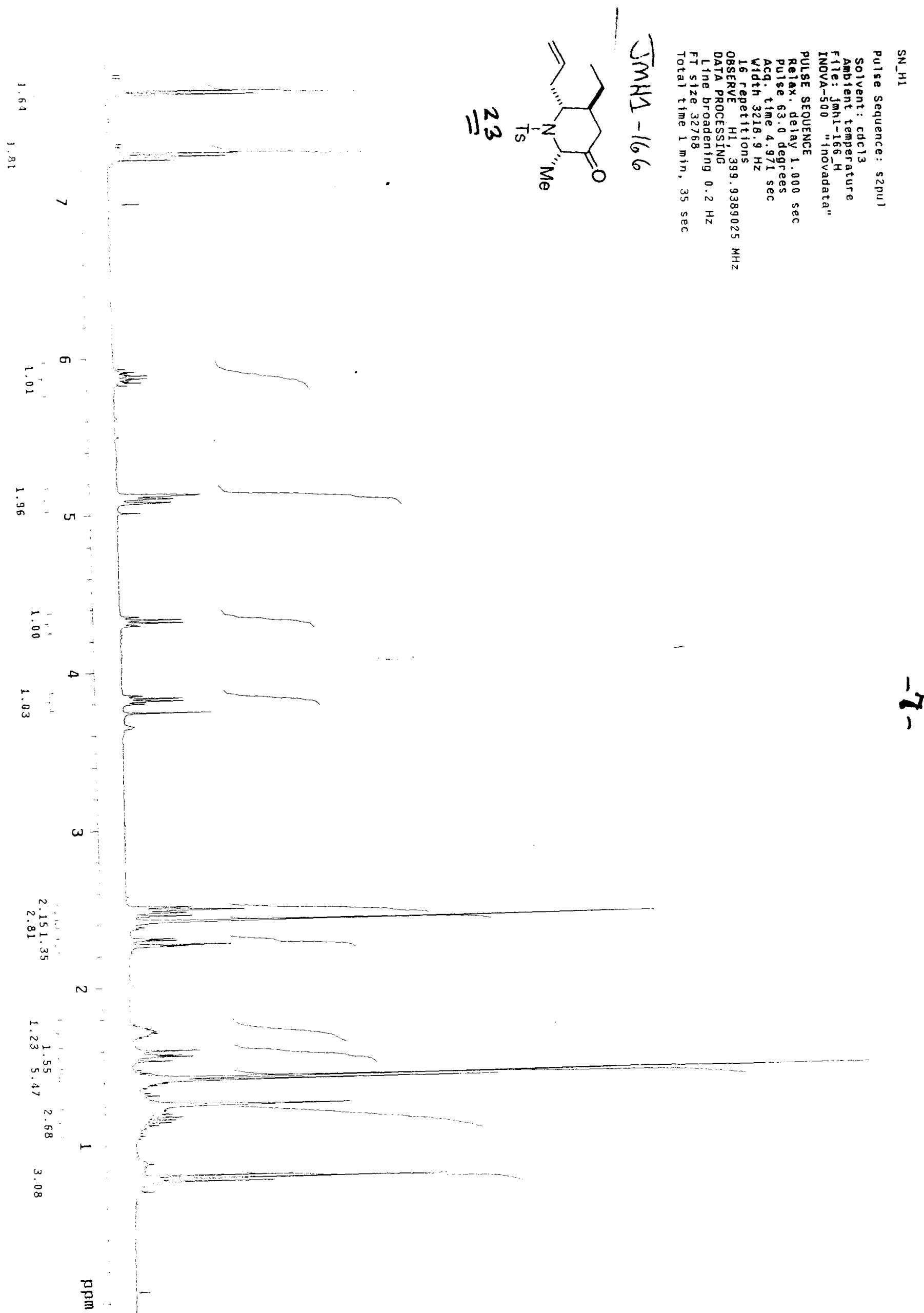


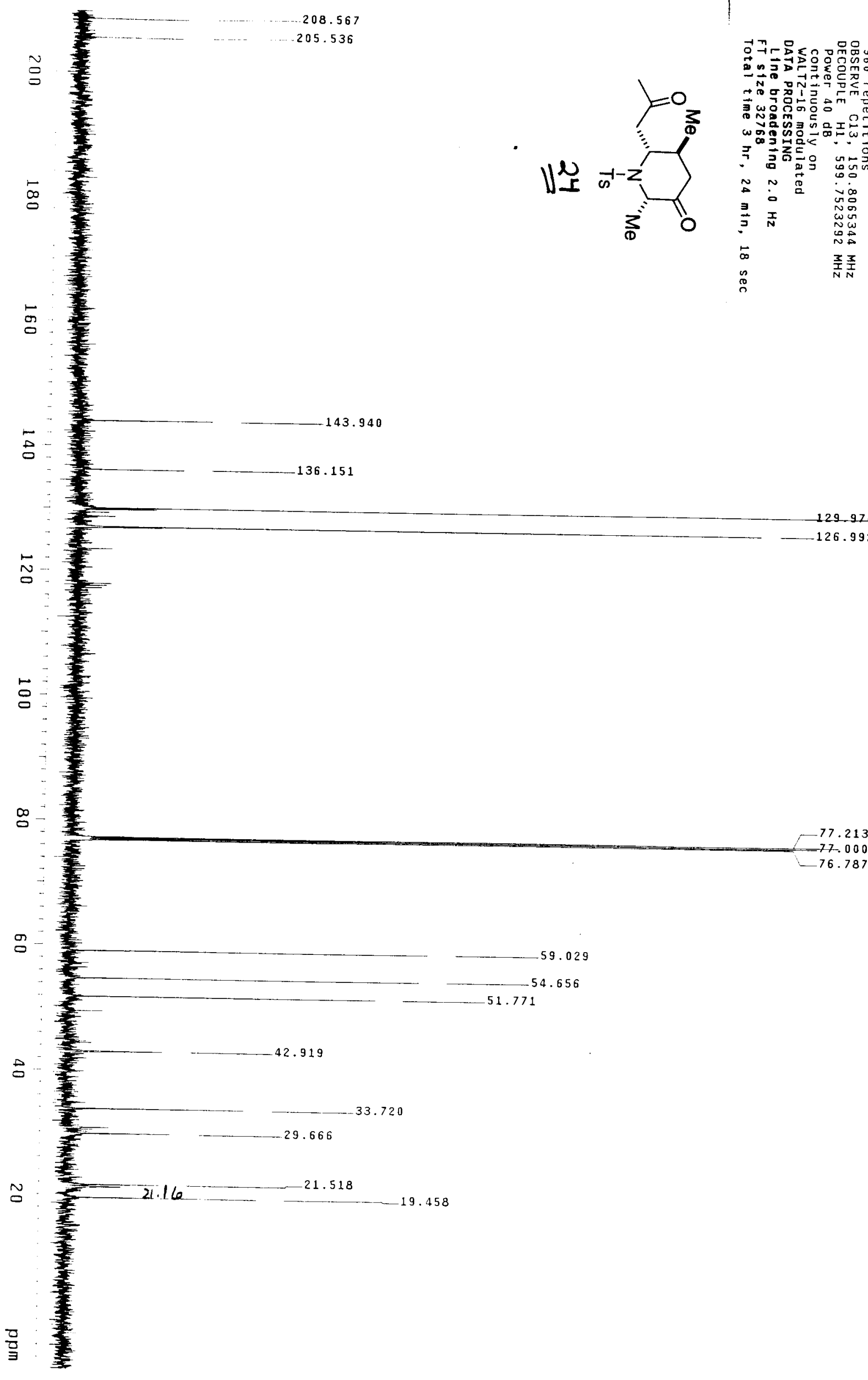


$\hat{\sigma}$

$i^{2}$

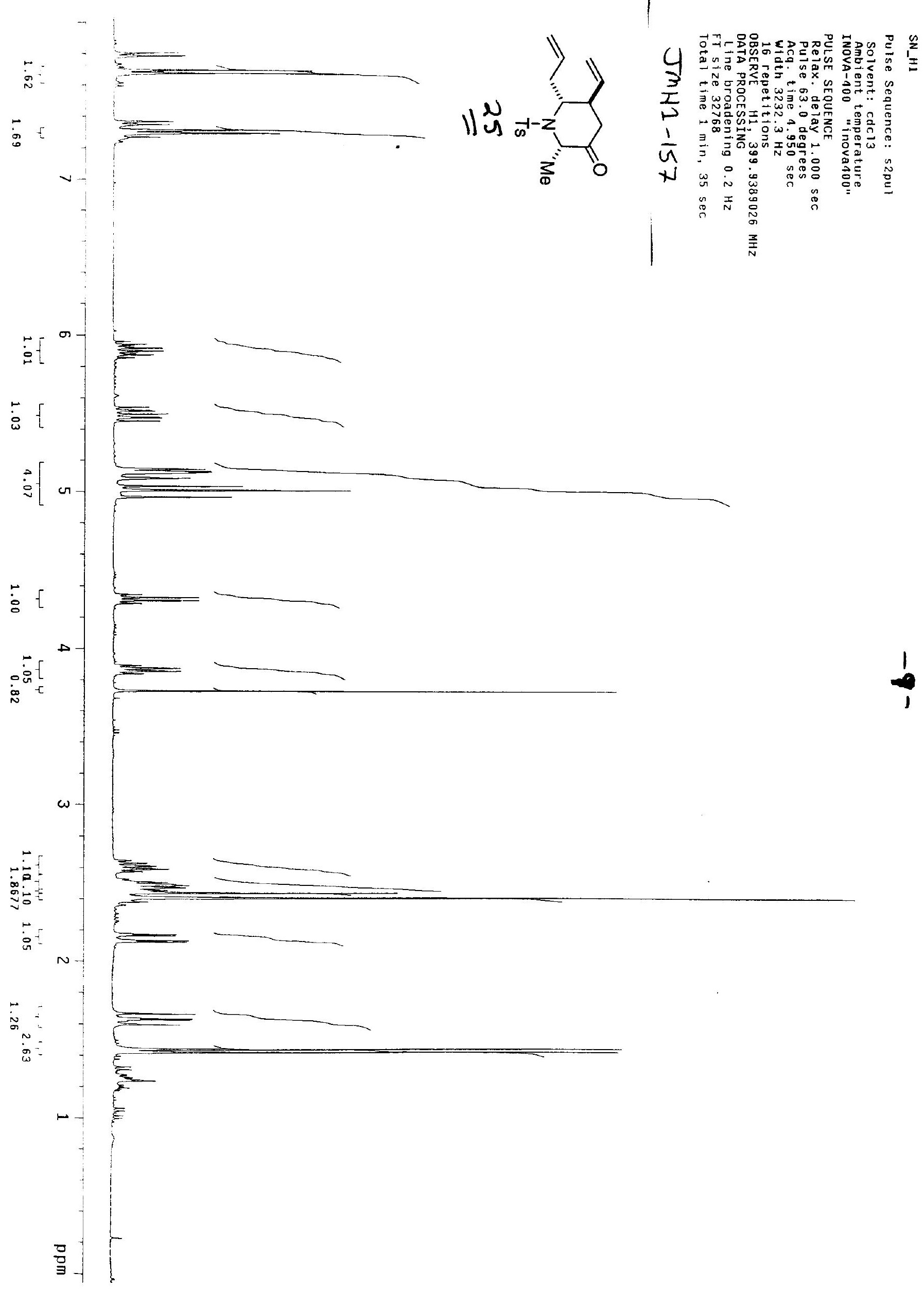



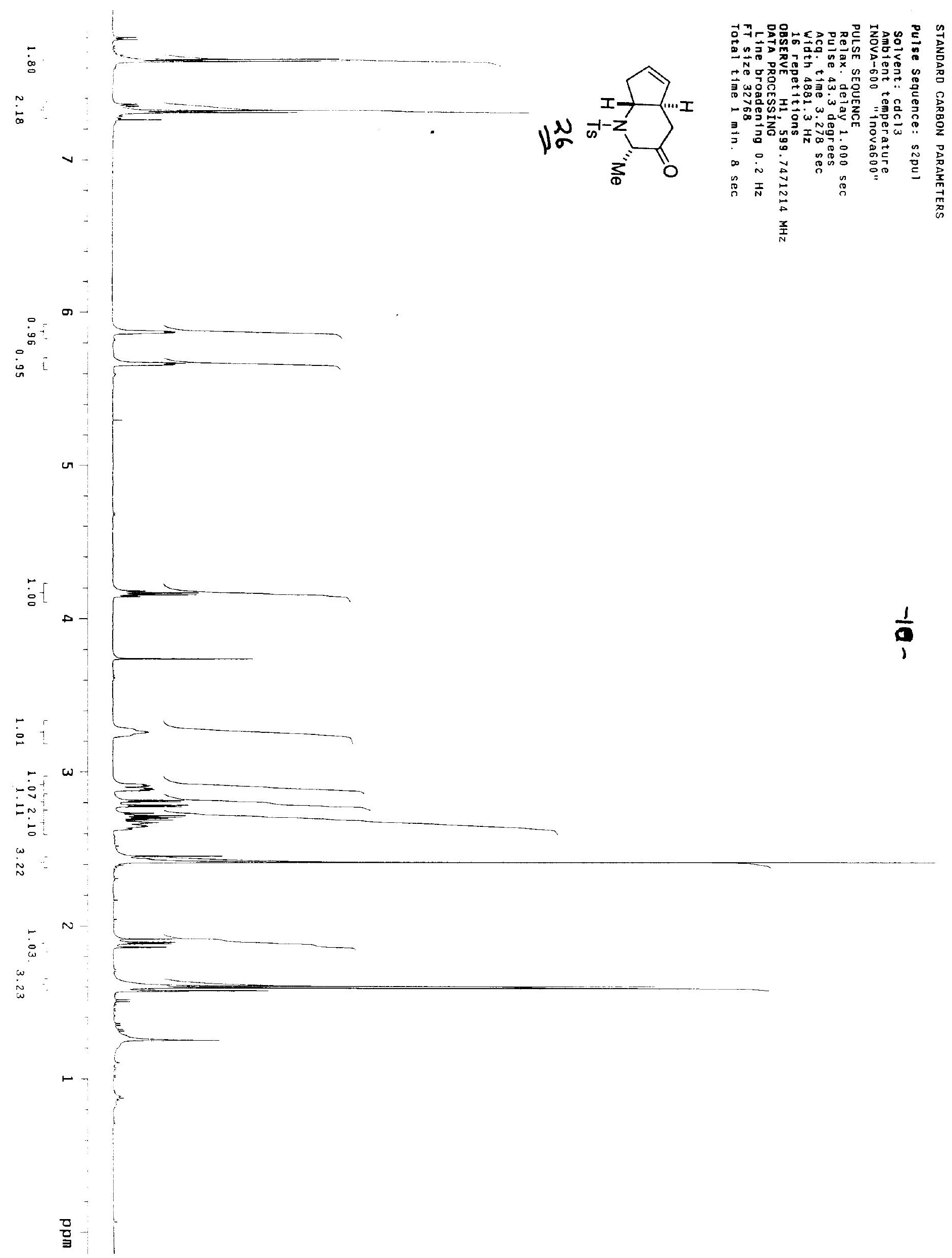


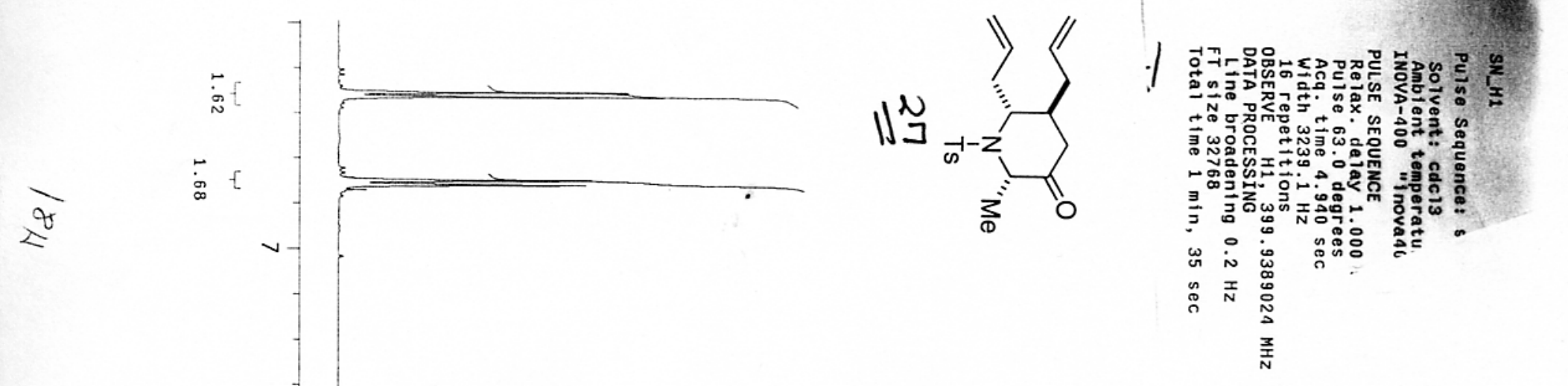




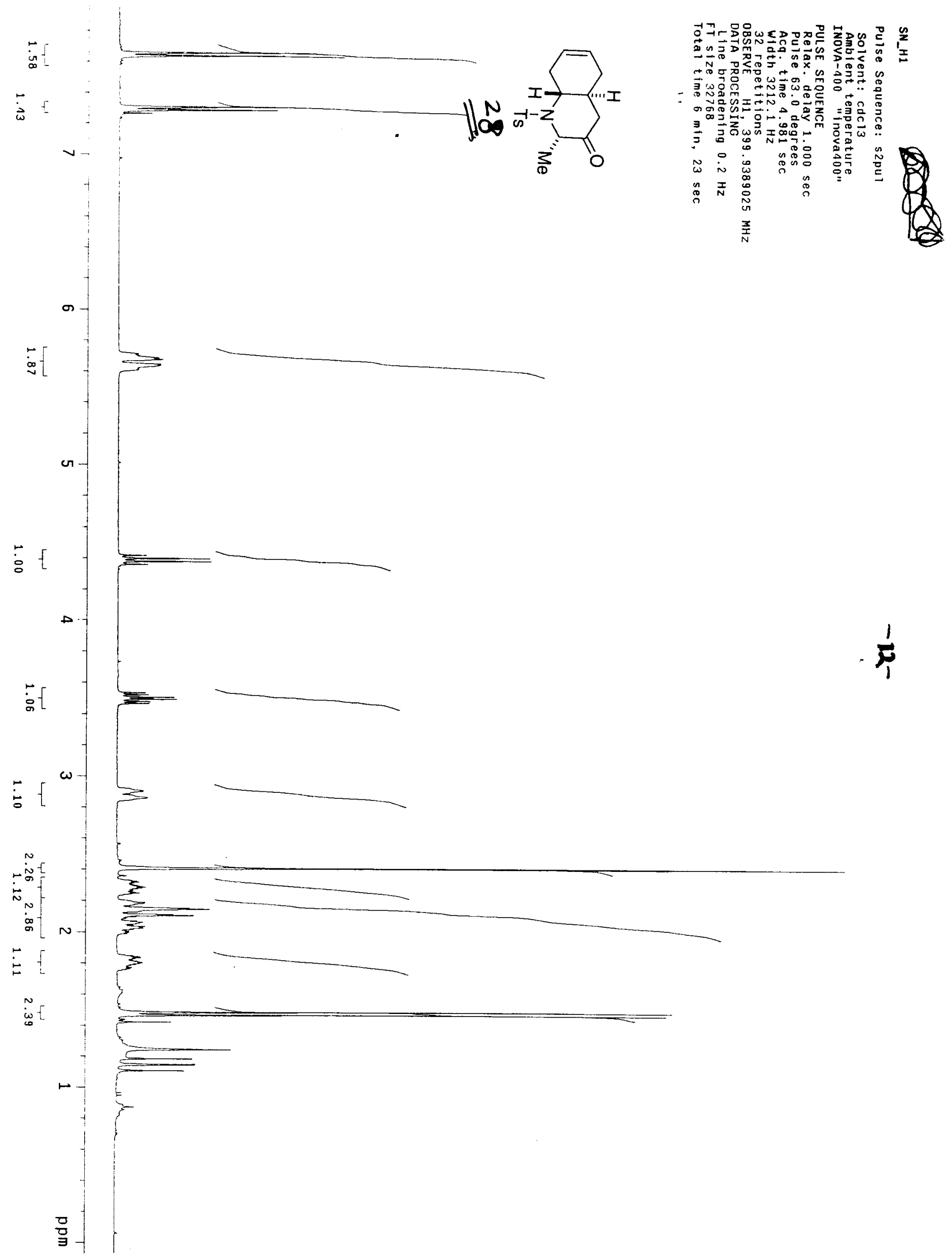




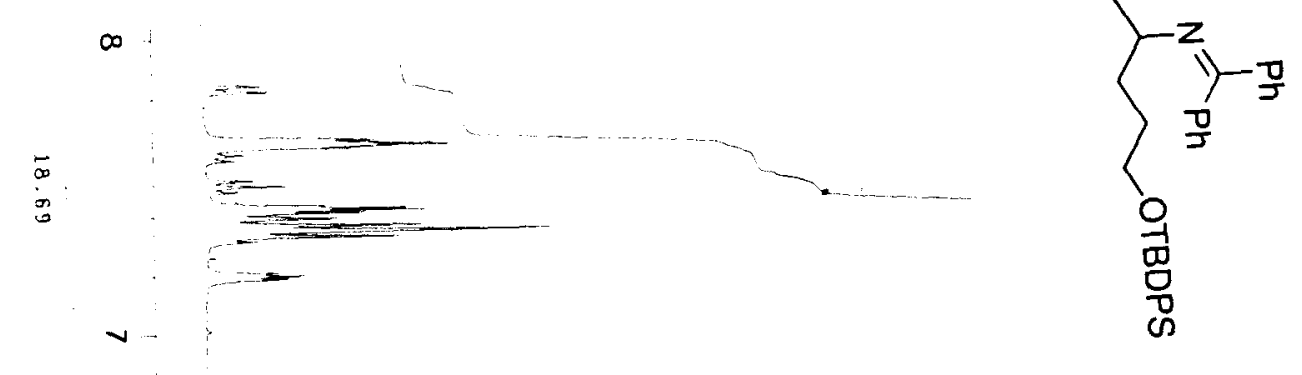

mumple

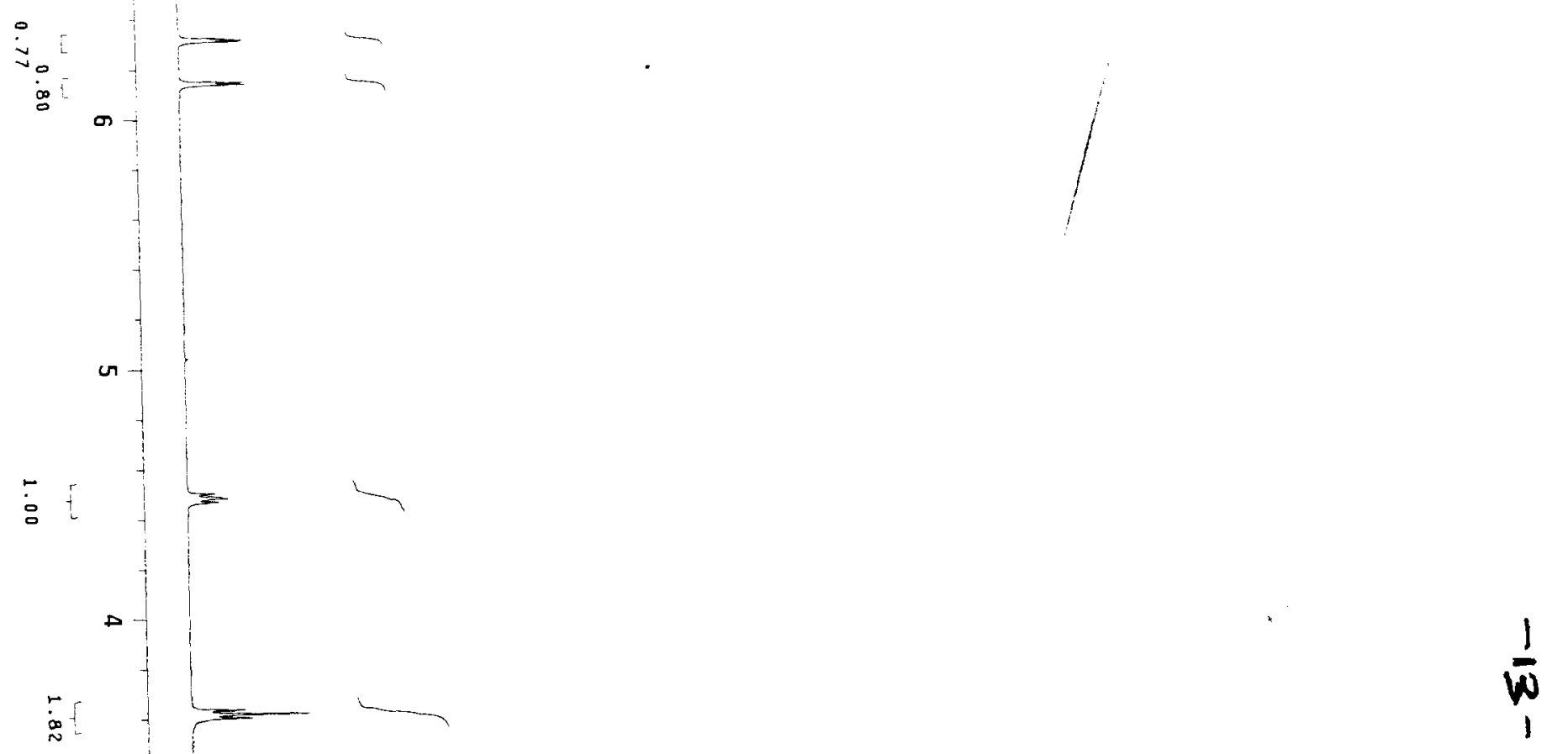

뫂 


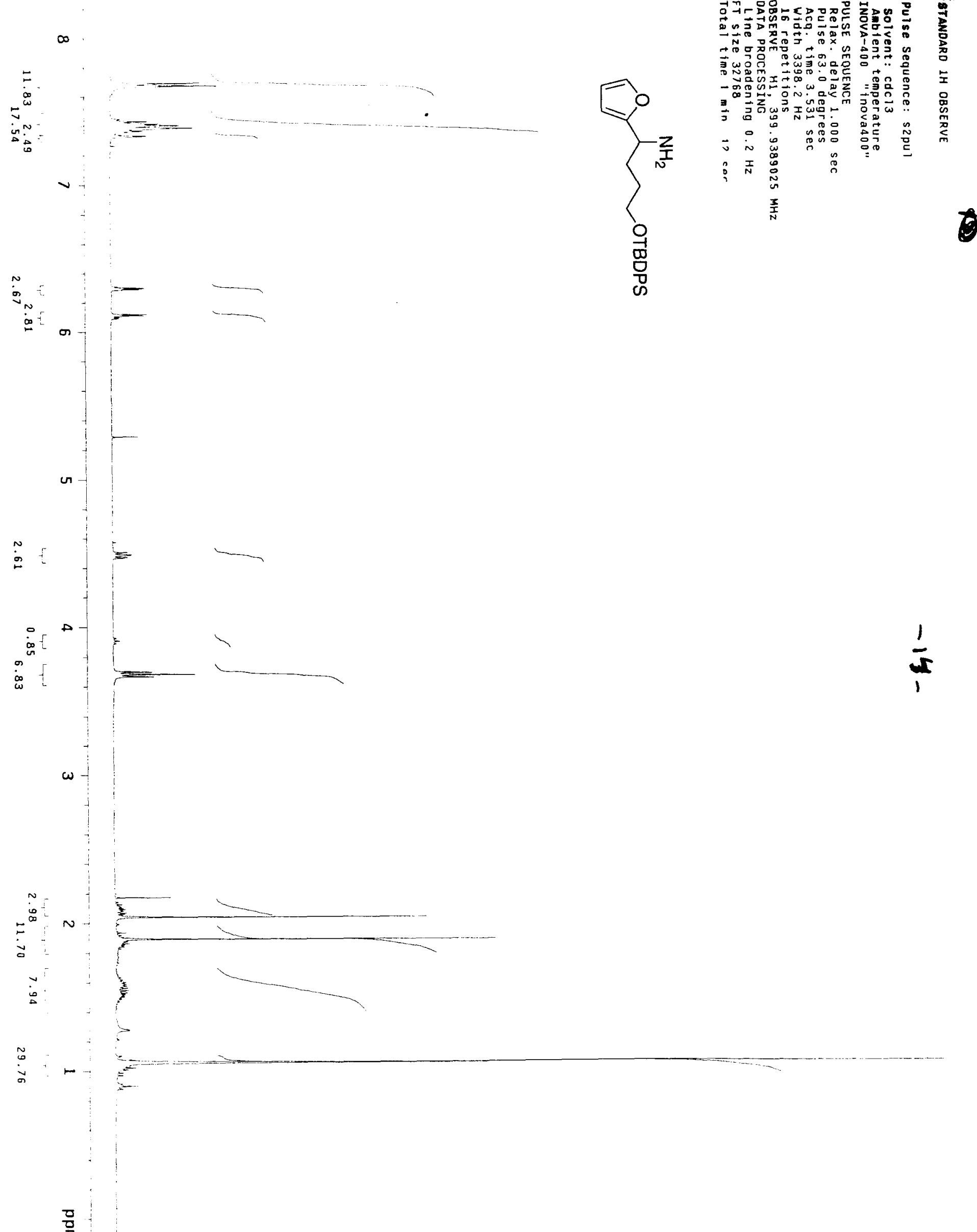




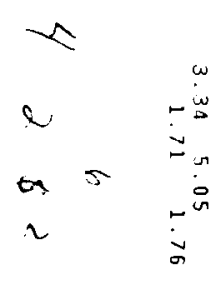
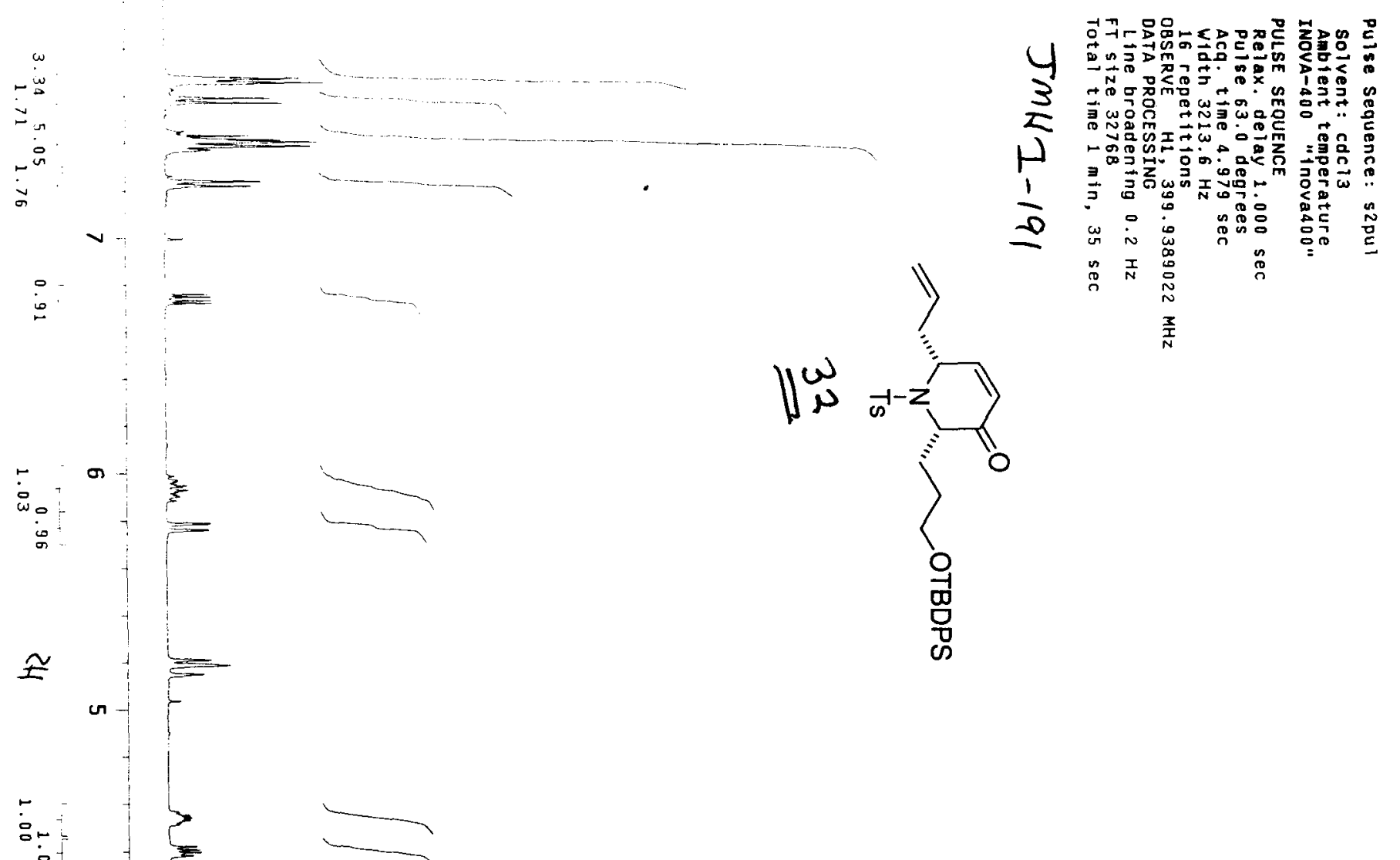

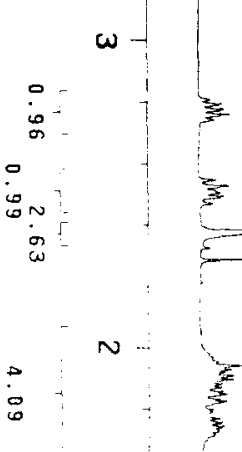

g.

몰 


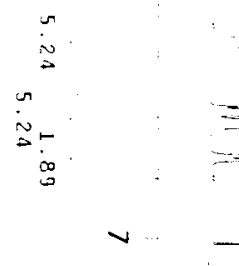

$\div-\sigma-$

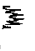

$\vec{\omega}_{\omega}=$

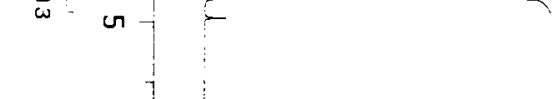

$\therefore+$

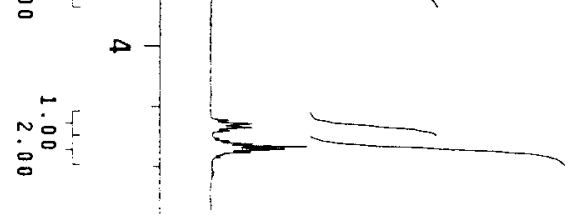

$\omega-$

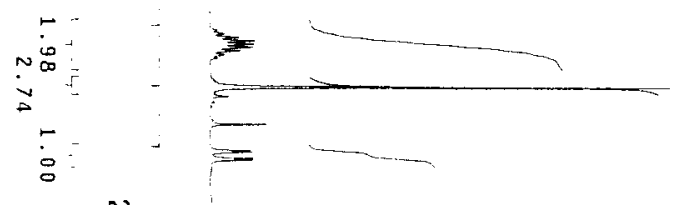

$\sim N \cdots$

$i_{\omega}+5$

in

$\omega$

?

5

$\stackrel{n}{\mathrm{~N}}$
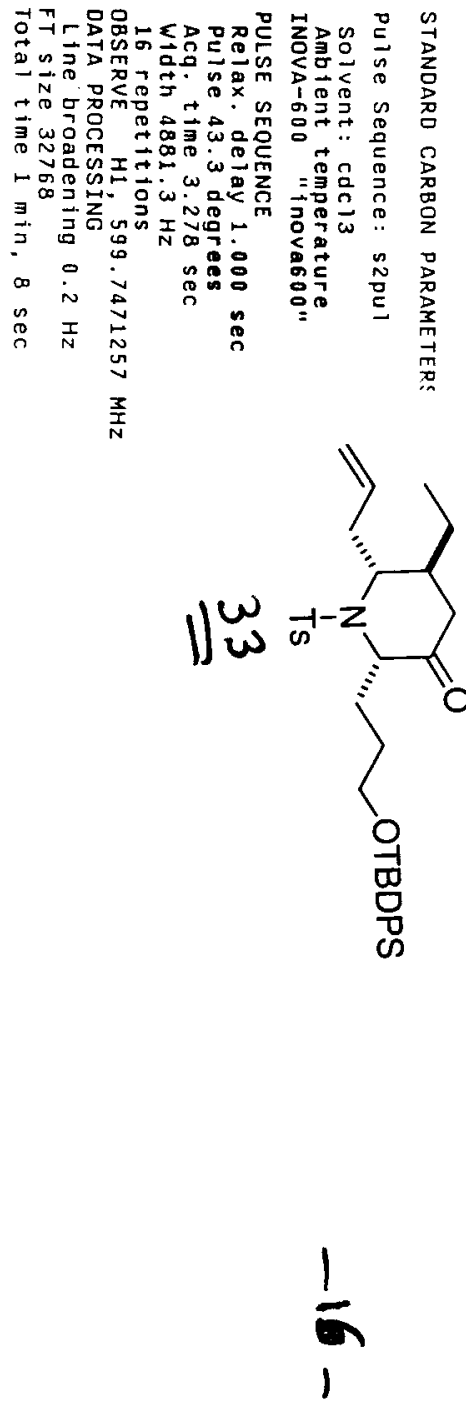

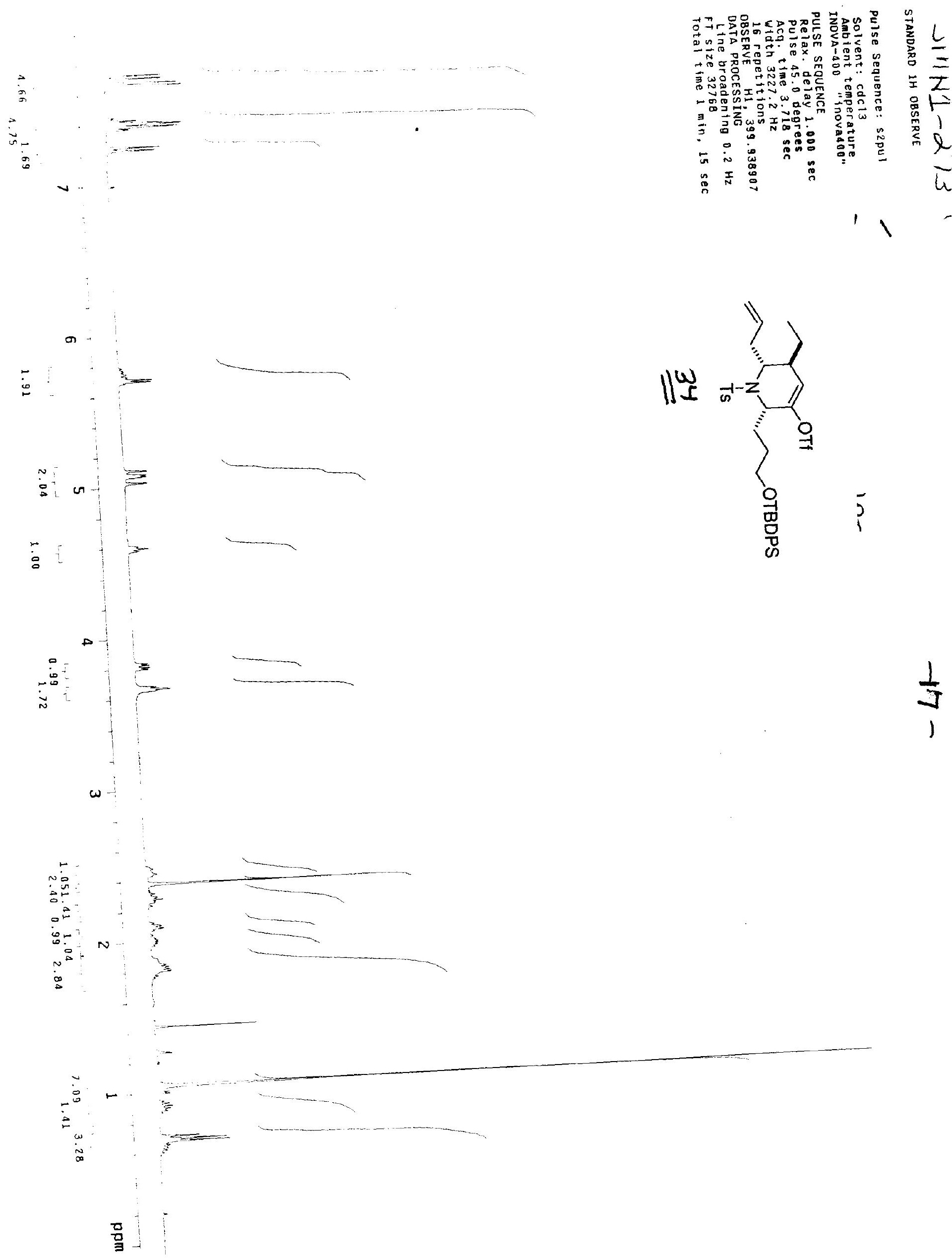
$\infty$

$\approx$
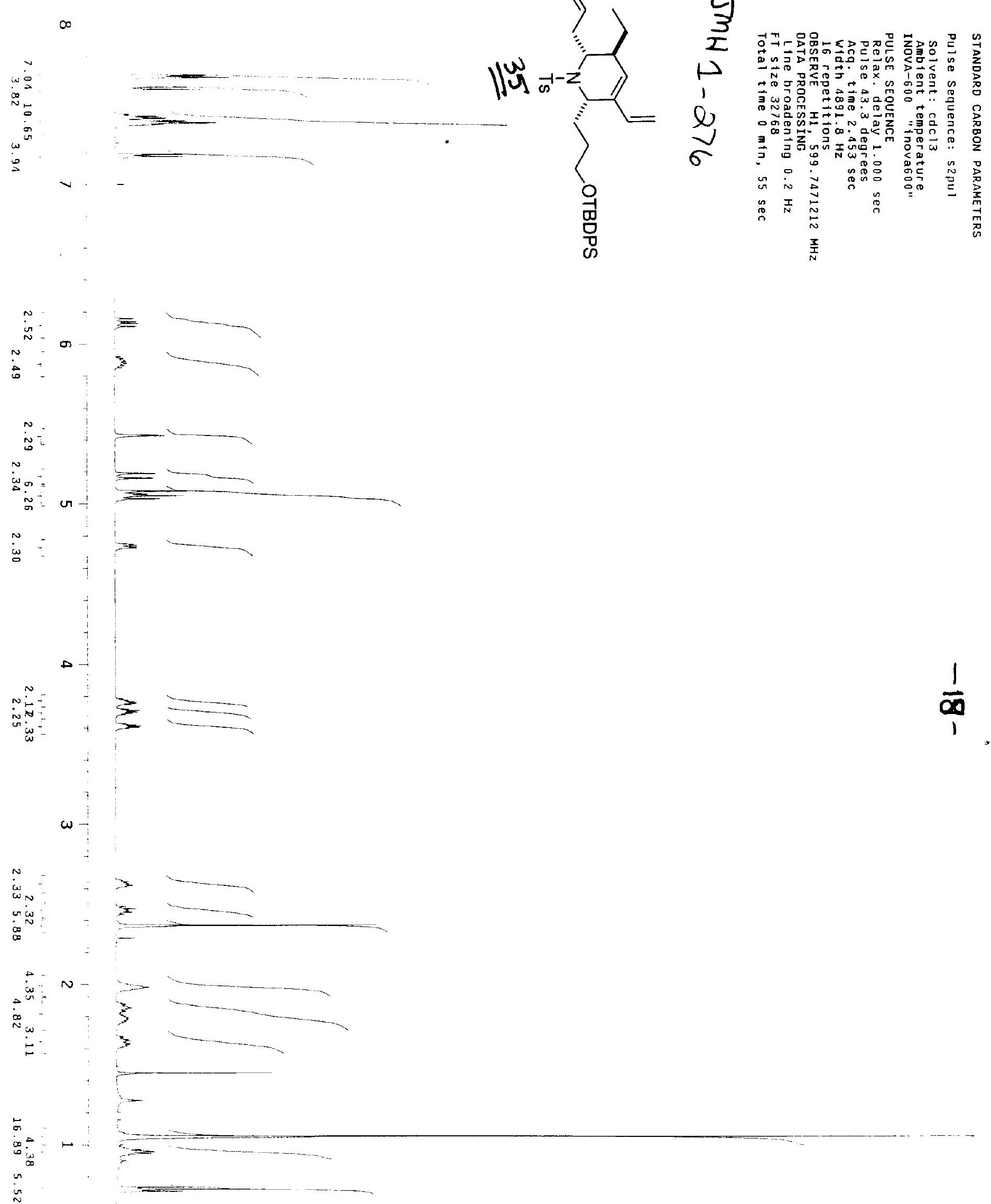
$\omega$

2
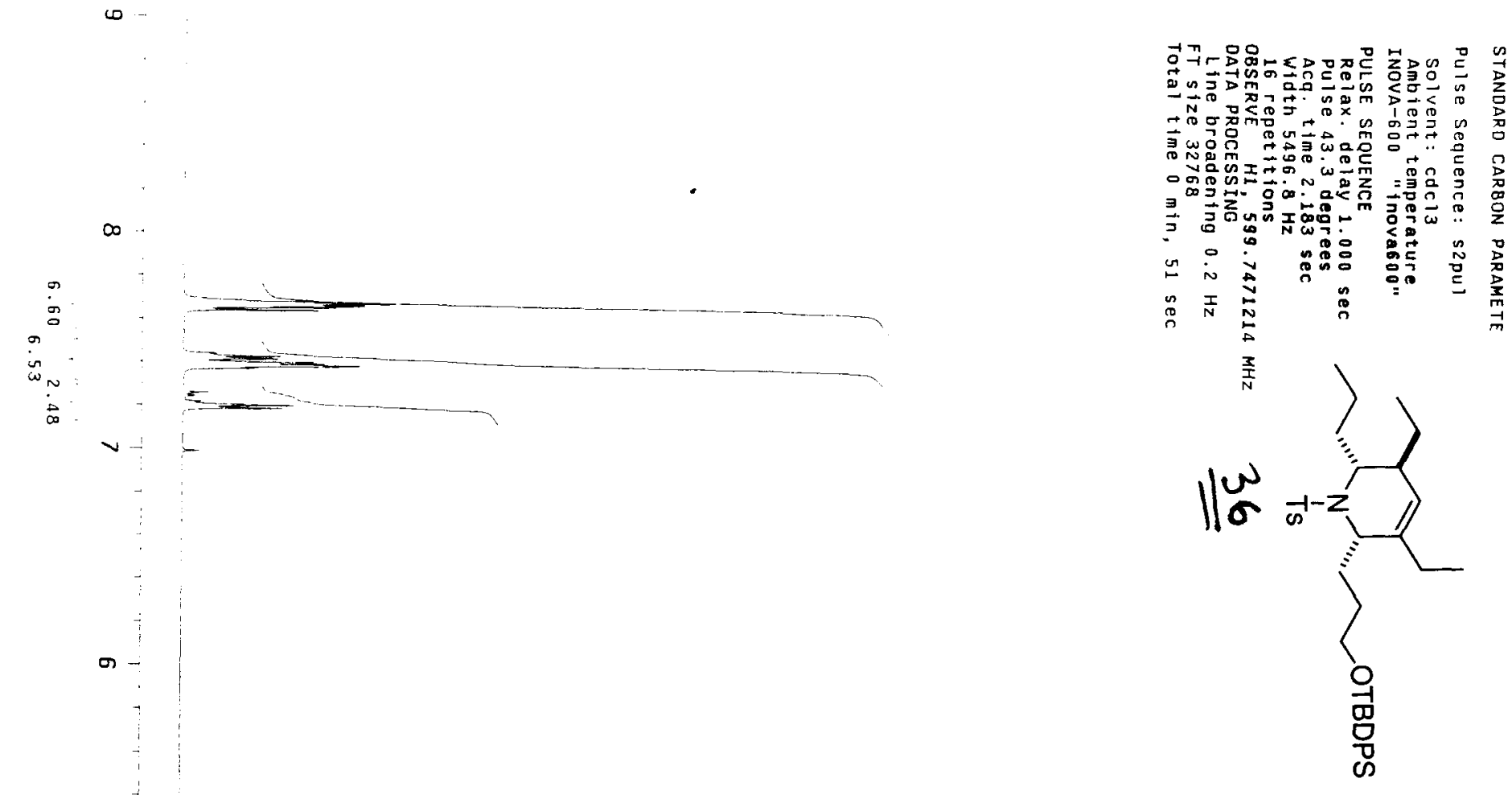

$\omega$

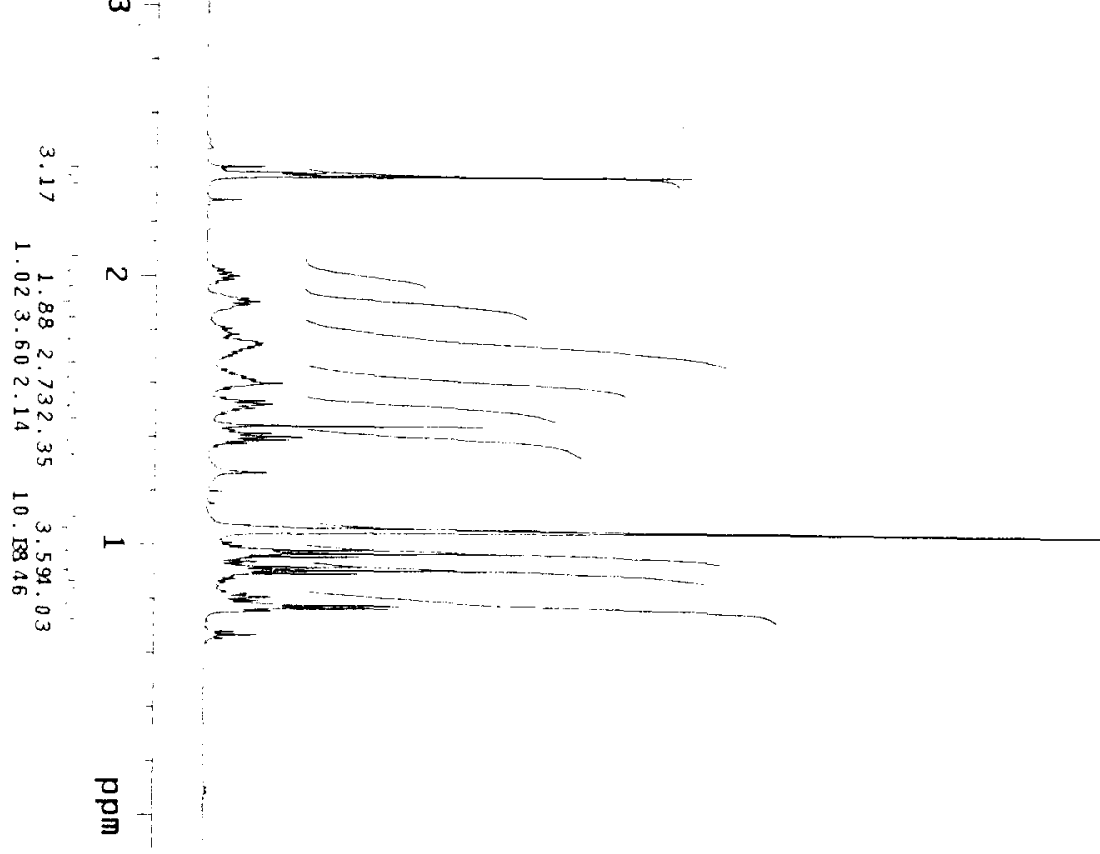



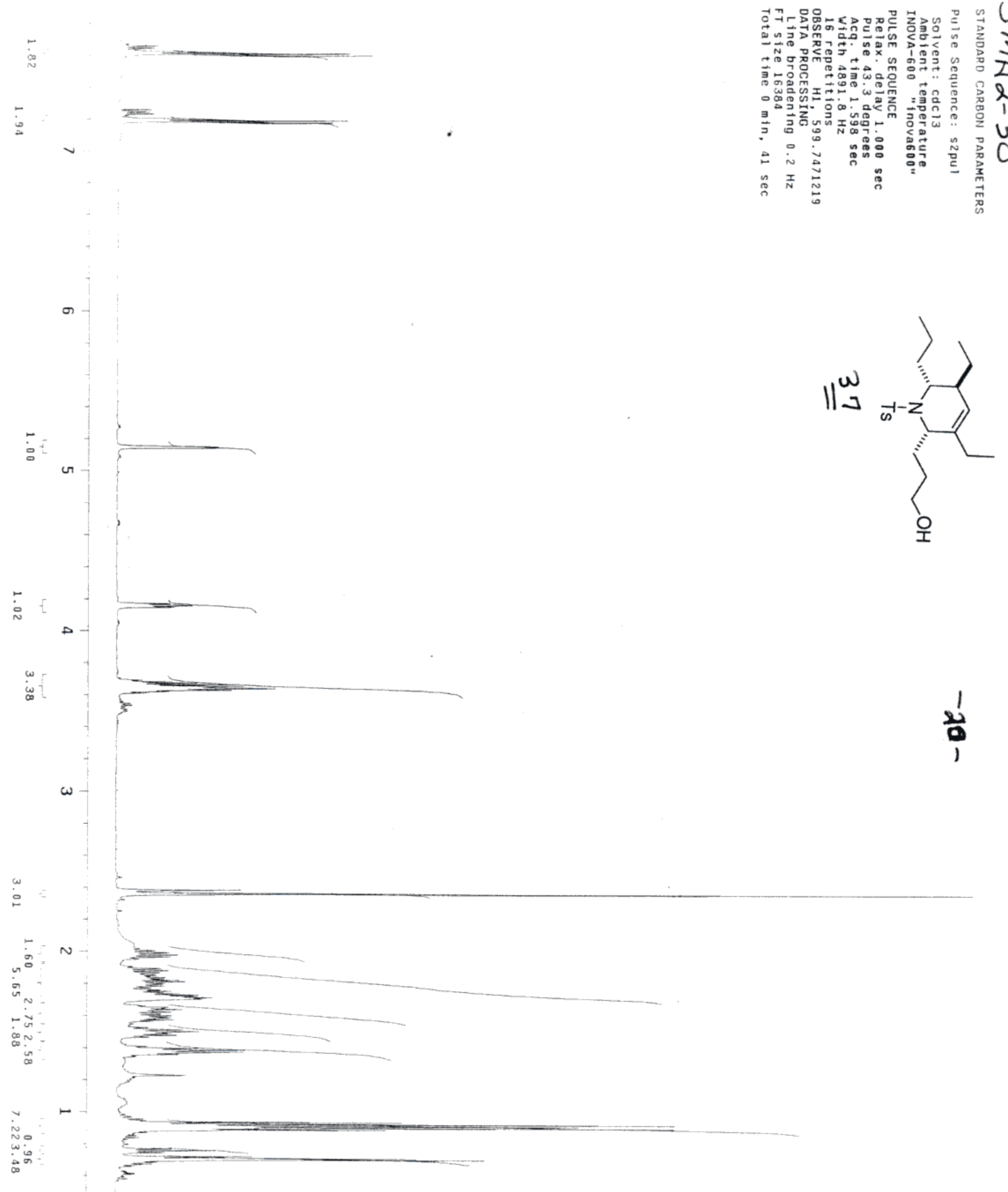

믐 


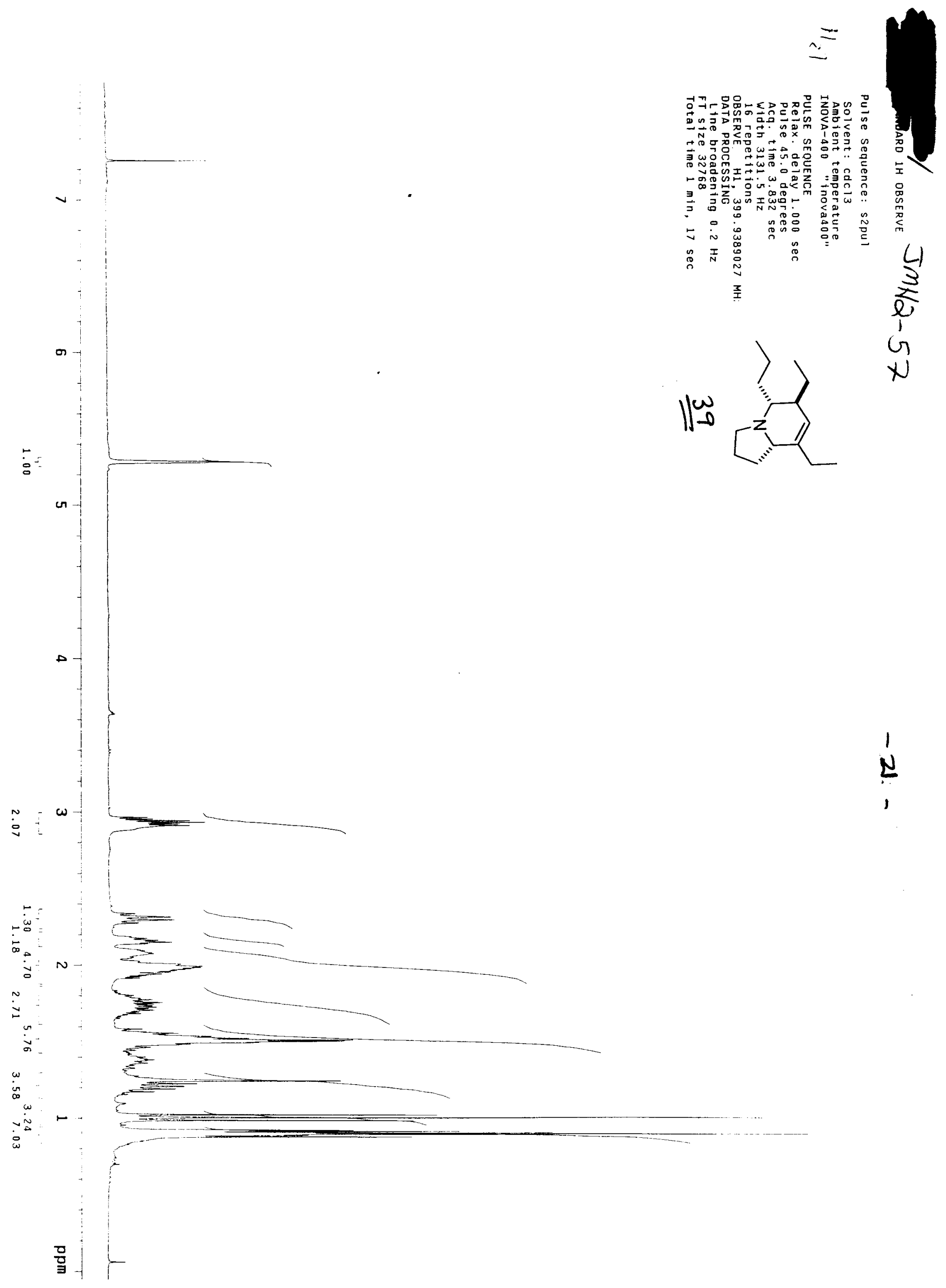




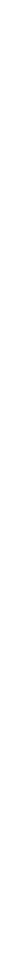

42.424

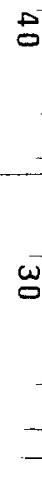

40.008

35.308

33.052

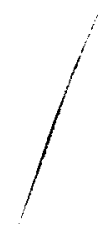

$-28.895$

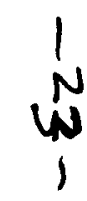

20.767

17.930

14.770 


\section{4}

Ortep Drawing of 6-Methoxy-2,5-dimethyl-1-(toluene-4-sulfonyl)-piperidin-3one (15).

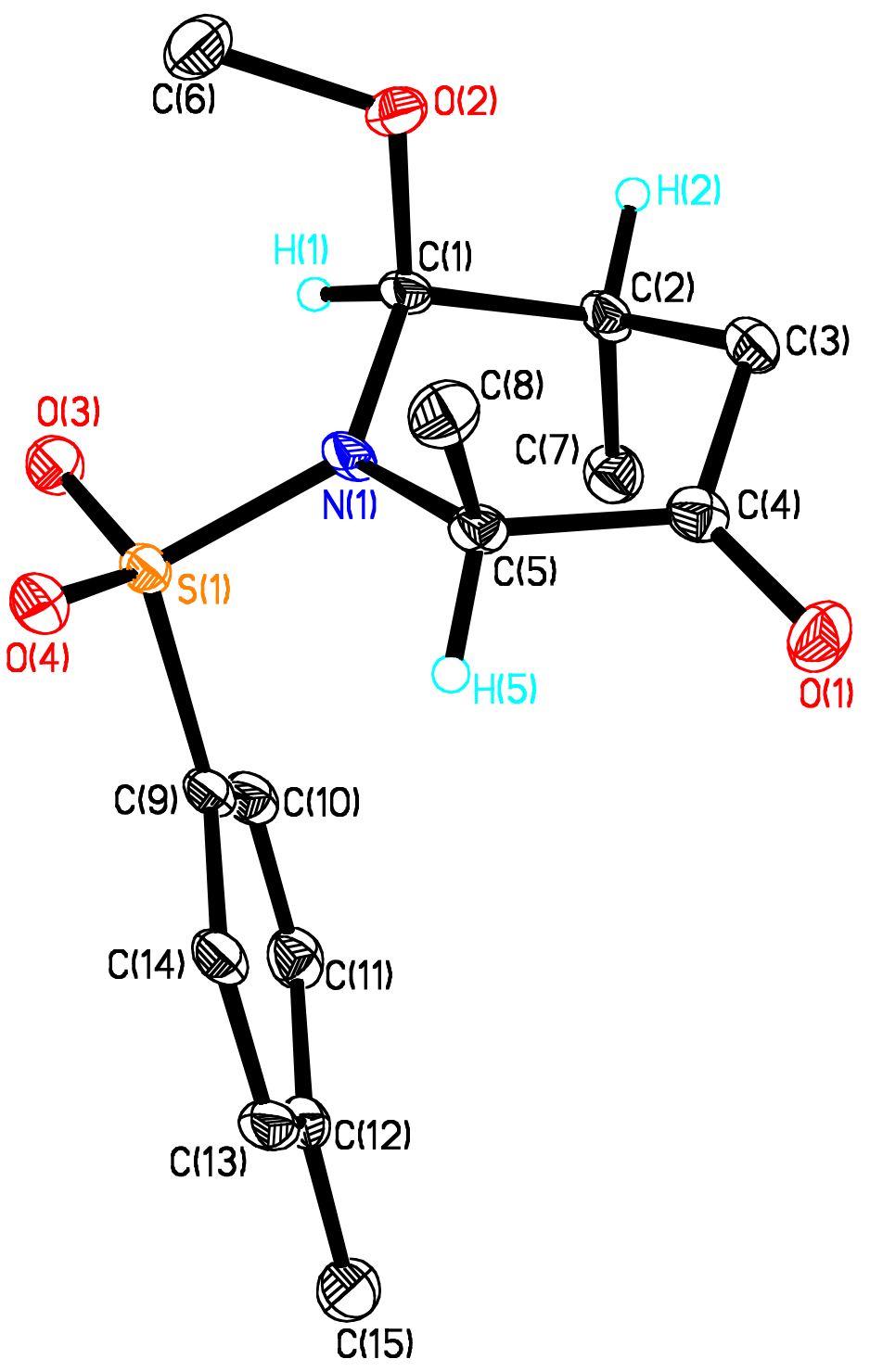

